\title{
Zur Notwendigkeit von Bildungsreformen
}

\author{
Jutta Allmendinger \\ Marcel Helbig
}

Nie zuvor war Bildung so wichtig wie heute. Eine gute Nachricht für alle, die eine gute Bildung erhalten durften. Eine umso schlechtere Nachricht für all jene, die nur wenig (aus-)gebildet sind. Dies gilt umso mehr, als viele von ihnen mehr hätten erreichen können, wären die Bildungschancen nicht so maßgeblich vom Elternhaus geprägt. Es ist höchste Zeit, dass die Zugangschancen zum Bildungssystem erhöht werden und die Bildungsarmut flächendeckend verringert wird. Die Voraussetzungen dafür sind heute besser denn je, da neben den leisen Rufen nach sozialer Gerechtigkeit der laute Ruf aus der Wirtschaft nach einer Bildungsreform erklingt.

\section{Bildungs(not)stand in Deutschland}

Bislang ging es bei der Diskussion um Inklusion und Exklusion im Bildungssystem oft um Fragen von Teilhabe und Gerechtigkeit. Bildung wurde als Bürgerrecht (Dahrendorf 1966) oder als Menschenrecht (Muñoz 2004) bezeichnet. Bildungssysteme wurden danach bewertet, wie selektiv sie sozial sind, also welche Chancen sozial Benachteiligte in einem Bildungssystem haben. Die Argumentationslinie von Pädagogik, Soziologie, Politikwissenschaft und Philosophie wird nun aber, wie bereits zu Zeiten des Sputnik-Schocks in den 1960er Jahren, von der Wirtschaft unterstützt. Bildung wird immer stärker zu einem Faktor, der über Wohl und Wehe einer Volkswirtschaft bestimmt - auch und gerade der deutschen. Zu diesem Ergebnis kam nicht nur die Expertenkommission für Forschung und Innovation (EFI) im Februar 2008, auch die OECD argumentierte so in ihrem „Wirtschaftsbericht Deutschland 2008“ (OECD 2008).

Die Begründung ist einfach. Infolge des Wandels der Wirtschaftsstruktur - weg vom primären und sekundären Wirtschaftssektor und hin zum wissensintensiveren tertiären Sektor - benötigt die deutsche Wirtschaft eine immer besser gebildete Erwerbsbevölkerung. Dieses „Mehr“ an Bildung wurde zu Zeiten der Bildungsexpansion zwischen 1950 und 1995 auch erreicht: Tatsächlich lag Deutschland 1994 mit einem Anteil von $23 \%$ der 25- bis 64-jährigen Bevölkerung mit tertiärer Bildung1 auch in der internationalen Spitzengruppe (IW 2007, S. 6). In den zehn Jahren zwischen 1994 und 2004 erhöhte sich dann der Anteil nur noch um zwei Prozentpunkte. Dies ist die geringste Steigerung aller OECD-Staaten: Deutschland ist innerhalb von zehn Jahren vom vierten auf den bescheidenen 14. Rang aller OECD-Staaten zurückgefallen. Die einstige Stärke ist zur Schwäche geworden, die Bildungsexpansion der 1970er und 1980er Jahre ist einer Bildungsstagnation gewichen.

Es brodelt. Deutsche Unternehmen überschlagen sich seit Monaten mit Hinweisen auf den bereits eingetretenen oder unmittelbar bevorstehenden Mangel an Fachkräften, Akademikern, insbesondere Ingenieuren (IW 2008; ZEW in BMBF 2007a). Dabei wird das Fehlen von gut (aus-)gebildeten Personen nach den Gesetzen von Angebot und Nachfrage dazu führen, dass Fachkräfte in Deutschland teurer werden - und damit auch die Produktionskosten für wissensintensive Güter und Dienstleistungen. Wissensintensive Branchen werden früher oder später darüber nachdenken müssen, in Länder auszuweichen, in denen Humankapital keine Mangelware ist - wie etwa in Schweden, Dänemark oder Finnland sowie im asiatischen Raum.

Das Fehlen von Humankapital ist der Punkt, an dem sich Wirtschaftswissenschaften und andere Disziplinen in ihren Analysen treffen. Eine Ausdehnung des tertiären Sektors ist heute allerdings nicht mehr durch eine Förderung von Kindern bildungsnaher Schichten zu steigern. Von Akademikerkindern nehmen bereits $83 \%$ ein Studium auf (BMBF 2007b, S. 111). Von den Kindern, deren Eltern nicht selber studiert haben, schaffen das nur $23 \%$. Hier liegen also hohe Potenziale für eine bessere Ausschöpfung brach, hier ist anzusetzen, wenn Bildungsgerechtigkeit erreicht werden will. So stark sich die Diskurse in den einzelnen Wissenschaften auch unterscheiden, ihre Folgerungen liegen eng beieinan- der: Gemeinsam fordern sie eine bessere Beteiligung sozial Benachteiligter an den höheren Bildungsgängen.

\section{Gesellschaftliche Inklusion durch Bildung}

Aus gerechtigkeitstheoretischer wie auch wirtschaftlicher Sicht stellt sich die Frage, wie man bislang benachteiligten Gruppen ein Mindestmaß an Bildung gewähren und zu höherer Bildung verhelfen kann. In Deutschland sind $20 \%$ der 15-Jährigen mit dem Stigma „kompetenzarm“ behaftet, sie erreichten bei PISA 2006 höchstens Lesekompetenzstufe I (OECD 2007), das „Existenzminimum". Auch erhalten $10 \%$ keinen und 32,2 \% eines Jahrgangs höchstens einen Hauptschulabschluss (StBa 2007, eigene Berechnungen). Diese Gruppe ist „zertifikatsarm“.

\footnotetext{
1 Genauer: Anteil von Personen mit akademischem Abschluss, Technikerabschluss oder Meisterabschluss an der 25-64-jährigen Bevölkerung eines Landes.
} 
Bildungsarmut ist mehr als eine Worthülse. Wer in diese Schublade kommt, der hat es schwer, in Deutschland eine Ausbildung zu finden. Er hat es damit auch schwer, eine „berufliche“ Tätigkeit zu finden, ist häufig von Arbeitslosigkeit betroffen und weist ein geringes Einkommen auf (Allen 2001). Bildungsarme haben aufgrund eines schlechten Gesundheitsverhaltens (Wolfe/ Zuvekas 1997; Max Rubner-Institut 2008) einen schlechteren objektiven und subjektiven Gesundheitszustand (Feinstein 2002; Wolfe/Zuvekas 1997). Bildungsarme sind anfälliger für Depressionen (Herzog et al. 1998). Vermittelt über das hohe Arbeitslosigkeitsrisiko leiden Bildungsarme häufiger an Mut- und Hilflosigkeit, mit mannigfachen Folgeproblemen; so zeigen sie etwa ein eingeschränktes Problemlösungsverhalten (Frese 1994). Ihre sozialen Beziehungen sind belastet, häufig kommt es zu einem sozialen Rückzug (Brinkmann/Wiedemann 1994).

Bildung ist der Schlüssel zur heutigen Wissensgesellschaft und wer diesen nicht besitzt, bleibt vor der Tür. Bildungsarmut ist soziale Exklusion, die den gesamten Lebensverlauf bestimmt.

Die Bildungsarmen sind dabei alles andere als eine heterogene Gruppe: Sie sind vor allem männlich, sie kommen aus Familien mit Migrationshintergrund und mit niedriger Bildung (Geißler 2005). Ungleiche Bildungsergebnisse gibt es in allen Bildungssystemen der Welt. Allerdings unterscheiden sich die Länder darin, wie stark der Zusammenhang zwischen Bildung der Eltern und der ihrer Kinder ausgeprägt ist. In Deutschland hängt Bildungserfolg so stark von der Bildung der Eltern ab wie in kaum einem anderen Land der OECD (OECD 2007). Auch wenn sich Politiker oft gegen Strukturdebatten verwehren (Der Spiegel 9/2008), soll im Folgenden gezeigt werden, dass (auch) Strukturreformen des deutschen Bildungssystems helfen würden, ausgeschlossene Gruppen zurück in die Gesellschaft zu holen, sie möglichst erst gar nicht zu verlieren.

\section{Soziale Ungleichheit}

Dass Bildung vererbt wird und wie das geschieht, wurde bereits in den 1960er Jahren gezeigt (Picht 1964), in den 1970er Jahren schlüssig erklärt (Boudon 1974) und seit- dem immer wieder empirisch nachgewiesen. Die Hauptargumente sollen hier wiederholt werden, um die potenzielle Wirkung von strukturellen Reformen erläutern zu können.

\subsection{FAMILIALE SOZIALISATIONS- BEDINGUNGEN}

Das Erreichen eines bestimmten Bildungszertifikates hängt in Deutschland vor allem davon ab, wie sich Kinder, Eltern und Lehrer an verschiedenen Bildungsübergangspunkten entscheiden. $\mathrm{Ob}$ das Kind nach der Grundschule auf die Hauptschule, die Realschule oder das Gymnasium geht, ob es nach dem Abitur eine Lehre macht oder studiert, wird stark durch den jeweiligen familiären Hintergrund beeinflusst. Für Deutschland sind diese Bildungsübergänge besonders entscheidend, weil sie kaum noch revidierbar sind. Eine Studie zur Durchlässigkeit des deutschen Schulsystems hat 2004 gezeigt, dass zwar viele Schülerinnen und Schüler bis zum 15. Lebensjahr die Schulart wechseln (14,4 \%), aber nur für 3,2 \% führt dies zu einem Bildungsaufstieg (Bellenberg et al. 2004).

Die Benachteiligung von Kindern bildungsferner Schichten und von denen aus Familien mit Migrationshintergrund - als einer Unterform oft bildungsferner Schichten - ist primären und sekundären Effekten zuzuschreiben (Boudon 1974). Primäre Effekte beziehen sich auf schichtspezifische Unterschiede im kulturellen Hintergrund und ihre Auswirkungen auf schulische Leistungen. Eltern beeinflussen über ihre Bildung und ihre kulturellen Güter - wie Bücher im Haushalt und das Vorhandensein von Musikinstrumenten - die Interessen und Kompetenzen ihrer Kinder. Dies geschieht bereits zu einem ganz frühen Zeitpunkt der kindlichen Entwicklung. Entscheidend ist, welches kulturell fruchtbare Umfeld Kindern geboten wird. Kulturelles Kapital wird in einem ständigen diffusen unbewussten Prozess vor allem von den Eltern, aber auch von Freunden und anderen Sozialisationsagenten (Stecher 2001, S. 145) auf die Kinder übertragen (Bourdieu 1983). Je umfassender ein Haushalt mit kulturellem Kapital ausgestattet ist - ob inkorporiert (kognitive und soziale Kompetenzen), institutionalisiert (Bildungszertifikate) oder objektiviert (kulturelle Güter) -, umso mehr kann davon auch an die Kinder weitergegeben werden. Kinder aus bildungsnahen Elternhäusern sind somit beim Erwerb von Kompetenzen im Vorteil.

Die Übertragung von kulturellem Kapital auf die Kinder beginnt schon kurz nach der Geburt. Eltern vermitteln ihren Kindern Wissen aus verschiedenen Bereichen, sie geben auch soziale Verhaltensweisen und Normen, Problemlösungskompetenzen und die Freude am Lesen und Hören weiter.

Eltern aus bildungsfernen Schichten können ihren Kindern meist weniger Wissen vermitteln und weniger Neugierde erzeugen. Oft fehlt es auch am Wissen um die Bedeutung von Bildung, häufig fehlt es schlicht an der Zeit, da beide Elternteile berufstätig sein müssen. Sie können ihren Kindern kaum leistungsbezogene Normen mit auf den Weg geben - weil sie diese selbst nicht leben. Bei Migrantenkindern kommt an dieser Stelle noch erschwerend hinzu, dass in der Familie teilweise nicht die Sprache gesprochen wird, die sie ab dem sechsten bzw. siebten Lebensjahr in der Schule benötigen werden und wie selbstverständlich sprechen müssen. Schon vor Schulbeginn werden so die zukünftigen Lebenschancen stark beeinträchtigt.

\subsection{FÖRDERMASSNAHMEN}

Chancengerechtigkeit zu erreichen, scheint relativ einfach. Kinder aus bildungsfernen Familien brauchen Hilfen. Geholfen werden kann auch außerhalb der Familie durch qualitativ hochwertige außerhäusliche Betreuung und Bildung vor der Einschulung.

Eine Vorschulpflicht, kombiniert mit professioneller Vorschulerziehung, würde Kindern aus bildungsfernen Schichten und Migrantenkindern helfen, ihre familiär bedingt geringen Chancen zu verbessern. Damit sind aber keine Verwahranstalten gemeint, sondern Einrichtungen, die von pädagogisch gut ausgebildetem Personal geleitet werden und mit Unterstützung von Sozialpädagogen auch auf individuelle soziale Probleme der Kinder eingehen können. Untersuchungen belegen deutlich, dass eine solche frühe Förderung von Kindern ökonomisch besonders sinnvoll ist. Je früher man also im Lebensverlauf mit der Förderung der Kinder ansetzt, desto weniger Geld muss später investiert werden, um benachteiligte Kinder kompensatorisch auf ein gewisses Bildungsniveau zu bringen (Wößmann 2006; Heckman/ Masterov 2007). 
Zusätzlich zur frühen Förderung von Kindern könnte man flächendeckend die Ganztagsschule einführen und so die Chancengleichheit weiter erhöhen und familiär bedingte Kompetenzdefizite ausgleichen. Vor allem für Kinder von Eltern, die kein anregendes kulturelles Umfeld bieten können, ist die Ganztagsschule vorteilhafter als das eigene Zuhause. Sie bietet die Möglichkeit, durch die längere Beschulung besser auf schwache Schüler einzugehen und sie innerhalb der Schule zu fördern. In der bisherigen Teilzeitschule ist individuelle Förderung kaum möglich. Sie muss teuer privat eingekauft werden. Das bevorteilt finanziell leistungsstarke und bildungsnahe Familien, die Nachhilfe auch deutlich stärker nachfragen (Schneider 2004), obwohl diese Kinder schon deutlich höhere Kompetenzen aufweisen. Derzeit kommt es zudem zu der paradoxen Situation, dass Eltern durch den Ausbau vorschulischer Kinderbetreuung eine Vollzeitbeschäftigung ermöglicht werden soll. Nach der Einschulung aber werden diese Kinder aufgrund von Halbtagsschulen am Nachmittag zu "Schlüsselkindern“ - oder ein Elternteil muss die Erwerbsarbeit wieder reduzieren.

Ein weiterer Grund für Ganztagsschulen ergibt sich aus der Verkürzung der Gymnasialzeit. Diese wird von tiefen Einschnitten in das Curriculum begleitet, nicht nur bei Fächern wie Musik, Ethik, Sport und Kunst. Doch selbst wenn die Reduktion nur diese Fächer betreffen würde, wäre es immer noch fatal, denn hier werden Schlüsselkompetenzen vermittelt, die für die Wissensgesellschaft wesentlich sind. Durch den Übergang zur Ganztagsschule würden Stunden gewonnen, die gerade diese Fächer stärken würden.

\subsection{WEICHENSTELLUNG BEIM SCHULÜBERGANG}

Dass Kinder aus bildungsnahen Schichten beim Kompetenzerwerb schon früh im Vorteil sind, belegt eine Vielzahl von Studien. Diese ungleiche Verteilung von Chancen setzt sich fort bis zum Übergang von der Grundschule ins dreigliedrige Schulsystem und auch darüber hinaus.

Den primären Ungleichheitseffekten folgen an den Bildungsübergängen die sekundären Effekte (Boudon 1974). Es geht um den Einfluss der sozialen Herkunft im Entscheidungsprozess beim Bildungsübergang. Kinder aus bildungsfernen Schichten und Migrantenkinder haben auch hier sys- tematische Nachteile. Hierfür gibt es vor allem drei Gründe, die am Beispiel der Entscheidung zwischen Gymnasium einerseits und Real- und Hauptschule andererseits verdeutlicht werden sollen. Der erste Hauptunterschied zwischen bildungsnahen und -fernen Schichten bei der Wahl zwischen Gymnasium und den beiden anderen Schulformen ergibt sich aus der höheren Bildungsdauer an Gymnasien. Für Eltern aus bildungsfernen Schichten, die im Durchschnitt ein geringeres Einkommen haben, ist das Gymnasium subjektiv und objektiv teurer. Gehen ihre Kinder auf ein Gymnasium, müssen sie länger versorgt werden. Zudem entstehen Opportunitätskosten, weil ihre Kinder erst später eigenes Geld verdienen. Der zweite Unterschied liegt in der unterschiedlich eingeschätzten Erfolgswahrscheinlichkeit des Gymnasialbesuchs. Eltern, die selber kein Abitur haben, können kaum einschätzen, ob ihr Kind das Gymnasium erfolgreich abzuschließen vermag. Der dritte Grund, der bildungsferne Schichten davon abhält, ihren Kindern einen Gymnasialbesuch zu ermöglichen, besteht in ihrer Schwierigkeit, den finanziellen und nichtmonetären Bildungsertrag der längeren Bildungsdauer $\mathrm{zu}$ bewerten.

Bildungsferne Schichten sind somit aufgrund ihrer Distanz zum Bildungssystem und mangelnder finanzieller Möglichkeiten im Vergleich zu bildungsnahen Schichten benachteiligt, wenn es darum geht, Kosten, Erfolgswahrscheinlichkeit und Nutzen des Gymnasialbesuchs ihrer Kinder einzuschätzen. Zudem wird in vielen Studien empirisch nachgewiesen, dass Kindern bildungsferner Schichten der Zugang zum Gymnasium auch objektiv erschwert wird: Sie müssen höhere Kompetenzwerte als Kinder bildungsnaher Schichten erzielen, um vom Lehrer für das Gymnasium empfohlen zu werden (Lehmann 1997; Bos et al. 2003). Hierbei sollte man Lehrerinnen und Lehrern allerdings keinen bösen Willen unterstellen (Ditton et al. 2005). Die Schulforschung zeigt, dass mit der Schichtzugehörigkeit typische Gesellschaftsbilder, Wertorientierungen, Erziehungseinstellungen und Erziehungspraktiken einhergehen. So wurden bei Kindern unterschiedlicher sozialer Herkunft auch Unterschiede im Sprachverhalten, im Wortschatz, beim Satzbau und im Abstraktionsvermögen festgestellt. Das gesamte Verhalten und der Habitus eines Menschen hängen erwiesenermaßen stark von seiner So- zialisation ab. Der Habitus von Kindern aus bildungsfernen Schichten oder mit Migrationshintergrund ist ein anderer als der von Akademikerkindern (Bourdieu 1983). Lehrerinnen und Lehrer können dies bei ihrer subjektiven Bewertung von Noten und Übergangsempfehlungen nicht ausblenden und bewerten Kinder aus bildungsfernen Schichten damit oft unbewusst schlechter. Eltern bildungsferner Schichten nehmen eine abschlägige Empfehlung für eine weiterführende Schulform in aller Regel zudem eher hin, manchmal korrigieren sie sogar die Empfehlung nach unten.

Ganz anders Eltern mit Abitur oder Hochschulabschluss. Sie akzeptieren selten eine Empfehlung für die Hauptschule oder die Realschule und setzen sich eher mit ihren eigenen Vorstellungen durch, und zwar ganz unabhängig von der Leistung ihrer Kinder (Ditton et al. 2005; Becker/Lauterbach 2004). Damit sind die theoretisch angenommenen Unterschiede beim Bewerten von Kosten, Nutzen und Erfolgswahrscheinlichkeit des Gymnasialbesuchs auch empirisch belegt.

Ungleichheiten im Bildungsübergang können ein gutes Stück weit abgebaut werden. Kein anderes westliches Industrieland trennt seine Schüler früher als Deutschland nach "gut" und „schlecht" (Hovestadt 2002). So müssen Eltern in Deutschland die Kosten, den Nutzen und die Erfolgswahrscheinlichkeit für das Gymnasium gleich für einen Zeitraum von acht Jahren bewerten. Lehrer haben im internationalen Vergleich die kürzeste Zeitstrecke, um die tatsächlichen Potenziale der Schüler erkennen zu können. Ein erhebliches Ausmaß von Fehlbewertungen durch Eltern und Lehrer ist damit wahrscheinlich. Dies ist umso gravierender, als diese weichenstellenden Entscheidungen im undurchlässigen deutschen Schulsystem so gut wie irreversibel sind (Bellenberg et al. 2004).

Ein Teil der Problemlösung liegt also darin, die gemeinsame Schulzeit zu verlängern. Mit jedem Jahr, um das die Trennung der Schulzweige verschoben wird, wird die Unsicherheit verringert bei der Voraussage, ob ein Kind tatsächlich das Abitur schaffen wird oder nicht. Mit jedem Jahr mehr in einer stärker heterogenen Schulform werden die Eltern und die Kinder durch andere Eltern und Kinder besser darüber informiert, wie hoch der Nutzen des Abiturs ist. Und eine aufgrund des späteren Übergangs ins Gymnasium verkürzte Gymnasialzeit verändert auch die Kostenkalkulation und 


\section{Abb. 1: Schüler mit allgemeiner Hochschulreife und Schüler auf För- derschulen, nach Bundesländern (2006) - in \% -}

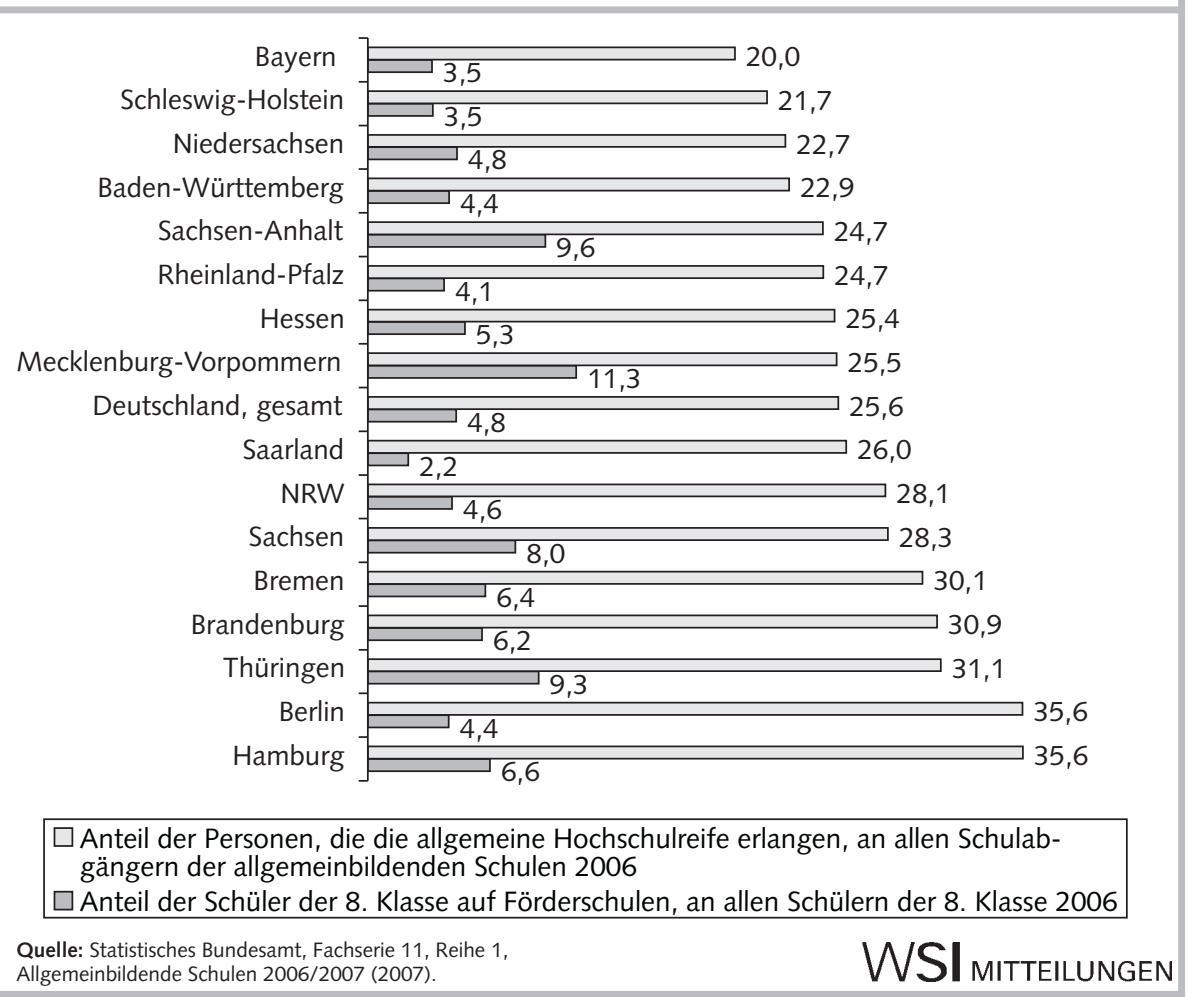

-bewertung positiv, die Eltern mit niedrigeren Einkommen vornehmen und vornehmen müssen. Zudem hat die Forschung gezeigt, dass vor allem benachteiligte Schüler in heterogenen Klassen besser lernen als in homogenen „Restschulen“, zu der die Hauptschule in manchen Teilen der Republik geworden ist (Turner 1964; Coleman 1966).

Vorschläge zur Einführung einer verpflichtenden Vorschule, einer flächendeckenden Ganztagsschule und einer späteren Trennung in verschiedene Schultypen sind alles andere als neu. In der Bildungsforschung ist es relativ unumstritten, dass diese Veränderungen die Chancengerechtigkeit im deutschen Bildungssystem erhöhen würden. Benachteiligte Gruppen, vor allem Kinder mit Migrationshintergrund und Kinder aus bildungsfernen Schichten, hätten eine höhere Chance, optimal gefördert zu werden. Bildungsarmut würde sich dadurch deutlich verringern und damit auch die Zahl der Jugendlichen, deren Möglichkeiten zu gesellschaftlicher Teilhabe später stark eingeschränkt werden. Ebenso würden die Transferzahlungen der Sozialkassen deutlich entlastet. Somit wäre auch ein Schritt hin zu einem „Sozialinterventionsstaat" getan. hen ist. Die Chance, die Hochschulreife zu erreichen, unterscheidet sich ebenso stark zwischen den Bundesländern wie das Risiko, die sogenannten Förderschulen zu besuchen.

In Bayern beenden nur knapp $20 \%$ eines Abschlussjahrgangs ihre schulische Ausbildung mit der Hochschulreife. Dagegen liegt die Wahrscheinlichkeit, die Schule mit der allgemeinen Hochschulreife abzuschließen, in fünf Bundesländern bei über $30 \%$, in Hamburg und Berlin sogar bei 35,6\%. Somit haben Schüler in Bayern, aber auch in Schleswig-Holstein (21,7 \%), Niedersachsen (22,7\%) und Baden-Württemberg $(22,9 \%)$ eine deutlich geringere Chance, ein Studium aufzunehmen. Verglichen mit den fünf Bundesländern mit hoher Abiturientendichte werden in den beiden südlichsten Bundesländern $10 \%$ bis $16 \%$ aller Schüler von der Hochschulreife und damit meist auch vom Studium ausgeschlossen. Dieser Sachverhalt entspricht nicht Unterschieden in der Leistungsfähigkeit der Schülerinnen und Schüler dieser Bundesländer. Im Gegenteil: Die Schülerinnen und Schüler Bayerns und Baden-Württembergs liegen im PISA-Vergleich der Bundesländer an der Spitze (PISA-Konsortium Deutschland 2004). Niedrige Abiturientenquoten gehen nicht mit niedrigen Kompetenzwerten einher, hohe Abiturientenquoten finden keine Entsprechung in durchschnittlich hohen kognitiven Kompetenzen. Diese Ungleichheiten gehen auf die unterschiedlichen Bildungsbzw. Schulsysteme in den Bundesländern zurück, auf die unterschiedlichen Schulgesetze und die unterschiedlichen normativen Vorstellungen, wie viele Menschen man an der höchsten Bildung teilhaben lassen und wie damit der Begriff der Bildungselite angepasst werden soll (Below 2002).

Im deutschen Föderalismus gibt es neben der Chancenungleichheit im Zugang zu höherer Bildung auch Unterschiede im unteren schulischen Bereich. In Deutschland besuchten 2006 durchschnittlich $4,8 \%$ aller Schüler der achten Klasse eine Förderschule. Das variiert ganz erheblich nach Bundesländern: In Bayern, dem Saarland und Schleswig-Holstein sind es deutlich unter $4 \%$, in den neuen Bundesländern bis zu 11,3\%. Auch hier kann gezeigt werden, dass es nicht dreimal so hohe Schülerinnen- und Schüleranteile mit Lern- und sonstigen Behinderungen in Sachsen, Sachsen-Anhalt, Thüringen und 
Mecklenburg-Vorpommern gibt wie in Bayern, dem Saarland oder in SchleswigHolstein. Auch hier führt der unterschiedliche Zuschnitt der Bildungssysteme und Schulgesetze dazu, dass in den ostdeutschen Bundesländern deutlich mehr Jugendliche gesellschaftlich ausgeschlossen sind und werden als in den westdeutschen Bundesländern: 77,2 \% der Förderschüler erlangen keinen Hauptschulabschluss (StBa 2007, eigene Berechnungen), sind also „absolut zertifikatsarm" und werden mit dieser schwerwiegenden Hypothek in der heutigen Wissensgesellschaft ein Leben lang belastet sein und dagegen anzukämpfen haben.

\section{Wechselwirkungen vertikaler und horizontaler Ungleichheit}

In Deutschland gibt es also nicht nur eine vertikale Benachteiligung von Kindern aus bildungsfernen Familien bzw. mit Migrationshintergrund, die von der allgemeinen Struktur des deutschen Bildungssystems gestützt und verstärkt wird. Es bestehen auch horizontale Chancenungleichheiten, die durch unterschiedliche Konzeptionen, Prämissen und Schulgesetze in den verschiedenen Bundesländern zu eigenen „Bildungssystemen" verfestigt worden sind. So zeigen sich je nach Bundesland deutlich ungleiche Chancen beim Zugang zu höherer Bildung. Auch kommt es in einigen Bundesländern zu einem wesentlich höheren Ausschluss von Kindern durch überproportional hohe Zuweisungen in Förderschulen.

Vertikale und horizontale Formen der Ungleichheit verstärken sich im deutschen Bildungssystem wechselseitig. So besuchen Förderschulen vor allem Jungen, Kinder mit Migrationshintergrund und aus bildungsfernen Familien. Kinder aus bildungsnahen Familien besuchen vor allem die Gymnasien. Je weniger Kindern eines Bundeslandes der Zugang zu einem Gymnasium gewährt wird, je geringer also dieses Bildungsangebot ist, desto höher fällt dort der Anteil von Kindern aus bildungs- nahen Familien aus, weil sie sich auf der Nachfrageseite gegen die bildungsfernen Schichten durchsetzen können. So werden Kinder aus bildungsfernen Familien in den Bundesländern systematisch stärker vom Gymnasialbesuch abgehalten, in denen anteilig die wenigsten Schüler zum Abitur kommen. Diese Konstellation ergibt sich auch aus dem PISA-Ländervergleich (PISA-Konsortium Deutschland 2004).

Diese beiden Formen von Ungleichheit im deutschen Bildungssystem sind nicht mehr legitimierbar, es bedarf tief greifender Reformen des deutschen Schulsystems. Diese Forderung ist nicht neu, sie wurde schon oft gestellt, geändert hat sich aber wenig. ${ }^{2}$ Könnte es in den kommenden Jahren anders werden?

\section{Es ging doch schon einmal}

Vielleicht schon. Denn neben der Forderung nach mehr Chancengerechtigkeit steht heute die rein wirtschaftliche Notwendigkeit der Reform. Dies betonte allerdings bereits Georg Picht in den 1960er Jahren mit der analogen Aussage, das deutsche Bildungssystem entspräche nicht mehr dem mittlerweile hoch industrialisierten Wirtschaftssystem (Picht 1964, S. 13). In nahezu sämtlichen Sektoren der gehobenen Berufe stellte er einen bedrohlichen Mangel an Akademikern und an mittleren Führungskräften fest: „Dieser Mangel wird in den nächsten Jahren einen Umfang annehmen, von dem sich kaum jemand eine Vorstellung macht, weil Staat und Wirtschaft noch nicht gelernt haben, die Schulstatistik in ihre Planungen und Berechnungen einzubeziehen" (ebd., S. 66). Der wirtschaftliche Aufschwung, so Picht, ist ohne qualifizierte Nachwuchskräfte nicht denkbar. Und weiter: „Wenn das Bildungswesen versagt, ist die ganze Gesellschaft in ihrem Bestand bedroht" (ebd., S. 16).

Schon damals gaben internationale Vergleichstudien der OECD Anlass, ein düsteres Bild der Zukunft Deutschlands zu zeichnen. Seinerzeit lag Deutschland in der vergleichenden Schulstatistik am unteren Ende der europäischen Länder - gemein- sam mit Jugoslawien, Portugal und Irland (ebd., S.16). Die von Picht beschriebene „Bildungskatastrophe“, auf die Deutschland zusteuerte, konnte abgewendet werden. Deutschland gelang bis Anfang der 1990er Jahre der Sprung zu einer der führenden Bildungsnationen. Nun stellt sich die alte Problemlage in der Wissensgesellschaft aufs Neue. Deutschland verliert im OECD-Vergleich seine Konkurrenzfähigkeit auf dem Bildungssektor und muss handeln. Es wird von allen westlichen Ländern ,abgehängt“. 1965 wurde der deutsche Bildungsrat als Reaktion auf Pichts Menetekel gegründet, um einen allgemeingültigen Reformfahrplan zu erarbeiten (Birsl/ Schley 2007, S. 7). Sicher waren Pichts Ausführungen nur eine Stimme aus dem Chor der Bildungsreformer nach dem SputnikSchock 1957, der schon das amerikanische Bildungssystem zu enormen Anstrengungen veranlasste. Aber diese Stimme hatte wohl nicht zuletzt wegen der auf das Wirtschaftssystem abstellenden Argumentation ein besonderes Gewicht.

Auch heute fordern zunehmend mehr Wirtschaftswissenschaftler tief greifende Reformen des deutschen Bildungssystems. Das bietet die Perspektive einer breiten Bildungsallianz. Viel Erfolg zu wünschen wäre den Politikwissenschaftlern, Pädagogen, Erziehungswissenschaftlern, Psychologen und Soziologen, die in den letzten Jahrzehnten Konzepte entwickelt haben, um sozial benachteiligten Gruppen bessere Voraussetzungen im Bildungssystem einzuräumen, ein gerechteres Bildungssystem zu schaffen und so wirkliche Chancengleichheit für alle, ungeachtet ihrer Herkunft, zu gewähren. Die Konzepte für eine umfassende Reform des Bildungssystems müssen nur noch aus der Schublade genommen werden, in der sie zu verstauben drohen. Die Chance für eine Reform war nie besser. Ergreifen wir sie?

Ausführungen zu der Frage, warum das deutsche Bildungssystem in den letzten Jahrzehnten nicht gerade durch Reformeifer bestimmt wurde, finden sich bei Brzinsky-Fay/Nikolai (2008) 
Allen, S. G. (2001): Technology and the Wage Structure, in: Journal of Labor Economics 19, S. 440-483

Becker, R./Lauterbach, W. (2004): Dauerhafte Bildungsungleichheit Ursachen, Mechanismen, Prozesse und Wirkungen, in: Becker, R./Lauterbach, W. (Hrsg.): Bildung als Privileg? Erklärung und Befunde zu den Ursachen der Bildungsungleichheit, Wiesbaden, S. 9-40

Bellenberg, G./Hovestadt, G./Klemm, K. (2004): Selektivität und Durchlässigkeit im allgemein bildenden Schulsystem. Rechtliche Regelungen und Daten unter besonderer Berücksichtigung der Gleichwertigkeit von Abschlüssen, Essen

Below, S. v. (2002): Bildungssysteme und soziale Ungleichheit. Das Beispiel der neuen Bundesländer, Opladen

Bundesministerium für Bildung und Forschung (BMBF) (2007a): Bericht zur technologischen Leistungsfähigkeit Deutschlands 2007, Bonn, Berlin Bundesministerium für Bildung und Forschung (BMBF) (2007b): 18. Sozialerhebung des Deutschen Studentenwerks. Die wirtschaftliche und soziale Lage der Studierenden in der Bundesrepublik Deutschland 2006 Berlin/Bonn

Birsl, U./Schley, C. (2007): Das Bildungssystem im „Kaukasischen Kreidekreis". Hemmnisse und Perspektiven in der Bildungspolitik, Hannover Boudon, R. (1974): Education, Opportunity and Social Inequality. Changing Prospects in Western Society, New York Bourdieu, P. (1983): Ökonomisches Kapital, kulturelles Kapital, soziales Kapital, in: Kreckel, R. (Hrsg.): Soziale Ungleichheiten, Bd. 2, Sonderband der Sozialen Welt, S. 183-198

Brinkmann, C./Wiedemann, E. (1994): Individuelle und gesellschaftliche Folgen von Erwerbslosigkeit in Ost und West, in: Montada, L. (Hrsg.): Arbeitslosigkeit und soziale Gerechtigkeit, Frankfurt/Main, New York, S. 175-192

Brzinsky-Fay, C./Nikolai, R. (2008): The Slow Introduction of New Public Management in the German Educational System. Why the Change from Politics to Management is still not popular? Präsentation bei ECPR Joint Sessions of Workshops, Rennes, 14. April

Coleman, J. S. (1966): Equality of Educational Oppurtunity, Washington D. C.

Dahrendorf, R. (1966): Bildung ist Bürgerrecht. Plädoyer für eine aktive Bildungspolitik, Hamburg

Ditton, H./Krüsken, J./Schauenberg, M. (2005): Bildungsungleichheit der Beitrag von Familie und Schule, in: Zeitschrift für Erziehungswissenschaft 2, S. 285-304

EFI (Expertenkommission für Forschung und Innovation) (2008): Gutachten zu Forschung, Innovation und technologischer Leistungsfähigkeit, Gutachten 2008, Berlin

Feinstein, L. (2002): Quantitative Estimates of the Social Benefits of Learning, 2: Health (Depression and Obesity), in: The Centre for Research on the Wider Benefits of Learning (Hrsg.): Wider Benefits of Learning Research Report 6, London, S. 1-52

Frese, M. (1994): Psychische Folgen von Arbeitslosigkeit in den fünf neuen Bundesländern: Ergebnisse einer Längsschnittstudie, in: Montada, L. (Hrsg.): Arbeitslosigkeit und soziale Gerechtigkeit, Frankfurt/Main, New York, S. 193-213

Geißler, R. (2005): Die Metamorphose der Arbeitertochter zum Migran- tensohn. Zum Wandel der Chancenstruktur im Bildungssystem nach Schicht, Geschlecht, Ethnie und deren Verknüpfungen, in: Berger, P. A./ Kahlert H. (Hrsg.): Institutionalisierte Ungleichheiten. Wie das Bildungswesen Chancen blockiert, Weinheim/München, S. 71-100

Heckmann, J. S./Masterov, D. V. (2007): The Productivity Argument for Investing in Young Children, IZA Discussion Paper 2725, Bonn Herzog, R. A./Hazel, R. M./Franks, M. M./Holmberg, D. (1998): Activities and Well-Being in Older Age: Effects of Self-Concept and Educational Attainment, in: Psychology and Aging 13, S. 179-185

Hovestadt, G. (2002): Was ist in Deutschland anders? Input-Indikatoren im internationalen Vergleich, in: GEW (Hrsg.): PISA ....und was in Deutschland anders ist. Ergebnisse - Analysen - Konsequenzen, Frankfurt Main, S. 67-83

Max Rubner-Institut, Bundesforschungsinstitut für Ernährung und Lebensmittel (2008): Nationale Verzehrs-Studie II. Ergebnisbericht, Teil I. Die bundesweite Befragung zur Ernährung von Jugendlichen und Erwachsenen, Karlsruhe

Muñoz, V. (2004): Economic, social and cultural Rights. The right to education, United Nations, Geneve

IW (Institut der deutschen Wirtschaft Köln) (2007): IW-Humankapitalindikator. Viel Wissen liegt brach, iwd 36, S. 4-5

IW (Institut der deutschen Wirtschaft Köln) (2008): Ingenieurlücke in Deutschland - Ausmaß, Wertschöpfungsverluste und Strategien, Köln Lehmann, R. H. (1997): Aspekte der Lernausgangslage von Schülerinnen und Schülern der fünften Klassen an Hamburger Schulen, Berlin Picht, G. (1964): Die deutsche Bildungskatastrophe. Analyse und Dokumentation in: "Christ und Welt" , Olten/Freiburg PISA-Konsortium Deutschland (Prenzel, M./Baumert, J./Blum, W./ Lehmann, R./Leutner, D./Neubrand, M./Pekrun, R./Rolff, H.-G./Rost, J./ Schiefele, U.) (2004): PISA 2003. Der Bildungsstand der Jugendlichen in Deutschland - Ergebnisse des zweiten internationalen Vergleichs, Münster Peisert, H. (1967): Soziale Lage und Bildungschancen in Deutschland, München

OECD (Organisation for Economic Co-operation and Development) (2007): PISA 2006. Science Competencies for Tomorrow's World, Paris OECD (Organisation for Economic Co-operation and Development) (2008): OECD Economic Survey Germany, Paris

Schneider, T. (2004) : Nachhilfe als Strategie zur Verwirklichung von Bildungszielen. Eine empirische Untersuchung mit Daten des Sozio-oekonomischen Panels (SOEP), Berlin

StBa (Statistisches Bundesamt) (2007): Allgemeinbildende Schulen Schuljahr 2006/07 Fachserie 11 Reihe 1, Wiesbaden

Stecher, L. (2001): Die Wirkung sozialer Beziehungen. Empirische Ergebnisse zur Bedeutung sozialen Kapitals für die Entwicklung von Kindern und Jugendlichen, Weinheim, München

Stuth, S. (2006): Regionale Disparitäten von Bildungsbeteiligung und mögliche Ursachen. Unveröffentlichte Diplomarbeit, Humboldt-Universität zu Berlin

Turner, R. H. (1964): The Social Context of Ambition, San Francisco Wolfe, B./Zuvekas, S. (1997): Nonmarket Outcomes of Schooling, in: Journal of Educational Research 6, S. 491-502 Wößmann L. (2006): Efficiency and Equity of European Education and Training Policies, Cesifo Working Paper 1779, München 